\title{
Comunicaciones romanas del ámbito provincial de Toledo en las antiguas fuentes itinerarias
}

\author{
G. Carrasco Serrano*
}

Sin duda alguna uno de los elementos más destacables en el fenómeno de la romanización, viene a estar representado por las vías de comunicación ${ }^{1}$, de ahi la cada vez mayor importancia y relieve que los estudios al respecto han llegado a adquirir últimamente.

En la actualidad la investigación sobre vías de comunicación romanas, presenta una complejidad derivada de la conjunción de trabajos de tipo arqueológico, histórico-geográfico y filológico que necesariamente requieren. Partiendo de este hecho, nuestro propósito en el presente estudio ha consistido básicamente en analizar aquellas vías y sus respectivas mansiones del área territorial correspondiente al ámbito provincial de Toledo, constatadas en las fuentes antiguas itinerarias, punto de partida obligado en toda investigación sobre viaria romana, dejando aparte por tanto, aquellos trazados no citados en dichas fuentes.

\section{ITINERARIO DE ANTONINO}

Viene a constituir sin duda y a pesar de los problemas que plantea, el Itinerario de Antonino ${ }^{2}$, la fuente antigua de carácter itinerario de más

\footnotetext{
* Universidad de Castilla-La Mancha.

J. M. ${ }^{a}$ BlÁzquez, "Causas de la romanización de Hispania", Mispania, 24, 1964, pp. 6 y 345 y ss.

2 Edición básica de esta obra ha sido la efectuada por P. WESSELING, Vetera Romanorum Itineraria, Amsterdam, 1735. Posteriores y más utilizadas son las de G. PARTHEY et M. PINDER, Itinerarium Antonini Augusti et Hierosolymitanum ex libris manuscriptis, Berlín, 1848, y la de O. Cuntz, Itineraria Romana, vol. I, Leipzig, 1929. Véase también K. MILLER, Itineraria Romana. Römische Reisewege an der Hand der Tabula Peutingeriana, Stuttgart, 1916; E. SaAvedRA, Discursos leidos ante la Real Academia de la Historia, Madrid, 1862; A. BLAZOUEZ, "Nuevo estudio
} 
relieve, por la importancia de los datos que proporciona. Son varias las vías de las mencionadas en esta obra, parte de cuyos trazados transcurrían por el área territorial de la actual provincia de Toledo, y más concretamente: Alio itinere ab Emerita Caesarea Augusta, Per Lusitaniam ab Emerita Caesarea Augusta, e Item a Liminio Toletum.

\subsection{Alio itinere ab Emerita Caesarea Augusta}

Es designada por E. Saavedra ${ }^{3}$ con el n. ${ }^{\circ} 25$, Alio itinere ab Emerita Caesaraugustam al igual que Wesseling: Constituia, como su propio nombre indica, una de las vías de las descritas por el Itinerario que unía a Emerita con Caesaraugusta. En este caso concreto y con una longitud total asignada de CCCXLVIII m.p., representaba la ruta más directa frente al Item ab Emerita Caesaraugusta (It. Ant., 433, 1-438, 1) con DCXXXII m.p., - la llamada Per Lusitaniam ab Emerita Caesarea Augusta (It. Ant., 443, 3$446,3)$, vias con las que presenta un recorrido común entre Titulcia y Caesaraugusta (It. Ant., 436, 1-438, 1; 438, 8-439, 4; 446, 1-446, 3). Por otra parte la falta de adecuación de las distancias proporcionadas por el Itinerario, ha llevado a plantear diversas hipótesis en un intento de dar solución satisfactoria a tales problemas, como la posibilidad de que la vía no partiese realmente de Emerita sino de una de las mansiones del camino de la plata, concretamente de Rusticiana (propuesta de A. BLÁzQuEZ) ${ }^{4}$, o incluso la posible omisión de alguna mansio como Lebura ${ }^{5}$ o Caesarobriga ${ }^{6}$. A dicha problemática cabe añadir, la no existencia de unanimidad por lo que respecta a la identificación segura de determinadas mansiones?

sobre el Itinerario de Antonino", BRAH, 31, 1892; ya más recientemente J. M. ROLdÁN, Itineraria Hispana. Fuentes antiguas para el estudio de las vias romanas en la Península Ibérica, ValladolidGranada, 1975.

3 Discursos..., op. cit, p. 74.

4 A. BlÁzQUez, MUSEA, 29,1920, pp. 23 y ss. Véase también J.M. Roldín, Iter ab Emerita Asturicam. El camino de la Plata, Salamanca, 1971, p. 158.

5 Véase al respecto $M$. FERnÁNDEZ MIRANDA et alii, "Alio itinere ab Emerita Caesaraugusta. La via romana entre Talavera de la Reina y Toledo y la implantación humana en el valle medio del río Tajo", Simposio sobre la red viaria en la Hispania Romana, Zaragoza, 1990, p. 157; M. FERNÁNDEZ MiRANDA et alii, "Indigenismo y romanización en la cuenca media del Tajo. Planteamiento de un programa de trabajo y primeros resultados", Actas del Primer Congreso de Arqueologia de la provincia de Toledo, Toledo, 1990, p. 17.

6 Vid. ME., 14, 1968, pp. 406-7; G. ARIAS, Repertorio de Caminos de la Hispania Romana, 1987, pp. $125-6$ y $367-8$.

7 Sobre esta vía en general, véase: F. CoELlo, "Vias romanas entre Toledo y Mérida", $B R A H, 15,1889$, pp. 5 ss.; A. BLAZQUEZ, "Informe relativo a la parte de la vía romana núm. 25 del Itinerario de Antonino", $B A A H, 60,1912, \mathrm{pp} .306-317 ; \mathrm{M}$. Roso DE LUNA, “Las vias romanas del 
Esta vía en su recorrido hasta Toletum, es descrita según la edición que hemos utilizado ${ }^{8}$, de la siguiente manera:

$\begin{array}{cll}\text { 438, } 2 & \begin{array}{l}\text { Alio itinere ab } \\ \text { Emerita Cae- } \\ \text { sarea Au- }\end{array} \\ 3 & \text { gusta } & \begin{array}{l}\text { m.p. CCCXLVIII, } \\ \text { sic: }\end{array} \\ 4 & \text { Lacipea } & \text { m.p. XX } \\ 5 & \text { Leuciana } & \text { m.p. XXIIII } \\ 6 & \text { Augustobriga } & \text { m.p. XII } \\ 7 & \text { Toletum } & \text { m.p. LV } \\ & \text {........... } & \text {........... }\end{array}$

\section{MANSIONES}

Aún cuando tan sólo Toletum se inscriba claramente en el ámbito que nos ocupa, la problemática en torno a Augustobriga para con el área objeto de estudio, hace conveniente sea igualmente contemplada.

\section{Augustobriga}

Además del Itinerario de Antonino, es mencionada también por otras fuentes antiguas como el Anónimo de Rávena $(312,12)$ con el nombre de

nordeste de Mérida”, BRAH, 60,1912, pp. 373-379; A. BLÁZQUEZ, MJSEA, 29, 1920, pp. 23-33; Id., MJSEA, 40, 1920-21, pp. 10-11; ME., 2, 1963; ME., 13, 1967; ME, 14,1968; J.M. ROLDÁN, Itineraria..., op. cit., pp. 86-87; G. ARIAS, Repertorio..., op. cit., pp. 125-6 y 367-371; ME, 17, 1988; ME, 18,1988; ME, 21,1989; $M E, 24,1989 ; M E, 27,1990 ; M$. FERnANDEZ Miranda et alii, "Alio itinere...", art. cit., pp. 155-163; M. FERnANDEZ MIRANDA et alii, "Indigenismo...", art. cit., pp. 15 ss.; ME, 34,1991; $M E, 35,1991 ; M E, 39-41,1992$; añádase y para el tramo entre Talavera de la Reina y Toledo, Y. ÁlvAREZ, "Aportación documental para el estudio del trazado de la vía romana entre Talavera de la Reina y Toledo", Actas de las Primeras Jornadas de Arqueclogía de Talavera de la Reina y sus tierras, Toledo, 1992, pp. 159-175; también y en relación con un miliario de Talavera, vid., D. URBINA, “Un miliario en Talavera de la Reina. Toledo", HAnt., XVII, 1993, pp. 349 ss.

s Itineraria Romana. Volumen Prius: Itineraria Antonini Augusti et Burdigalense, Edidit $O$. Cuntz, Leipzig, 1929. En el aparato crítico de esta edición se hace constar: 438,2 , itenere $L$ caesarea, ea sup. Iin. IL; 5 leutiana $B ; 6$ augustobrica $B$.

9 E. SaAvedra, Discursos..., op. cit., p. 74 proporciona la distancia de mpm XXII. 


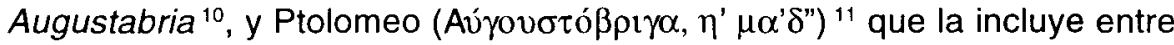
los núcleos vettones. Por su parte Plinio cataloga a los augustobrigenses como estipendiarios (IV, 118: stipendiariorum quos nominare non pigeat, praeter iam dictos in Baeticae cognominibus, Augustobrigenses...) ${ }^{12}$, Ilegando con posterioridad a alcanzar, probablemente, categoría municipal ${ }^{13}$.

No totalmente resuelta su identificación, J. A. Cean Bermúdez ${ }^{14}$ al igual que M. Cortés y López ${ }^{15}$ y P. Madoz ${ }^{16}$ la llegarían a situar en la localidad de Villar del Pedroso, de donde procede la inscripción funeraria de Flavia Rufina augustobrigensis recogida en CIL, II, $941^{17}$. Sin embargo para A. Blázquez se situaría en el emplazamiento de Vascos ${ }^{18}$, al oeste de la provincia de Toledo, en el término municipal de Navalmoralejo, hipótesis seguida igualmente por J. M. Roldán ${ }^{19}$. También y de forma más mayoritaria, en base al testimonio epigráfico de Talavera la Vieja en el que se hace mención del Senatus Populusque Augustobrigensis ${ }^{20}$, se ha

${ }_{10}$ Augustobriga constituye uno de los diversos topónimos constatables en la Península con sufijo en -briga, vid., en este caso, A. HoLdER, Alt-Celtischer Sprachschatz, I, Leipzig, 1896, p. 286; en las variantes del elemento -briga y en dichas formas toponimicas según las distintas fuentes, se encuentra frecuentemente -bria, véase al respecto $M^{a}$ L. ALBERTOS FIRMAT, "Los topónimos en -briga en Hispania", Veleia, 7, 1990, pp. 131-144; también sobre dichos topónimos y su distribución en el territorio hispano J. UNTERMANN, Sprachräume und Sprachbewegungen in vorrömischen Hispanien, Wiesbaden, 1961, pp. 13 ss. y map. 3.

1 Ptol., II, 5, 7.

12 C. Plint Secund, Naturalis Historiae, edidit C. Mayhoff, Lipsiae, MCMVI.

13 Véase R.K McELDERRY, "Vespasian's reconstruction of Spain", JAS, 8, 1918, p. 73; H. GALSTERER, Untersuchungen zum römischen Städtewesen auf der iberischen Halbinsel, Berlín, 1971, $p$. 53; A. TovaR, Iberische Landeskunde. Die Völker und die Städte des antiken Hispanien, II. Lusitanien, Baden-Baden, 1976, p. 235; sobre el testimonio de la tribu Quirina en $C / L, I I, 928$ y 5343 vid., R. WIEGELS, Die Tribusinschriften des römischen Hispanien, Berlin, 1985, p. 73; también y en general sobre la problemática de esta comunidad en relación a su carácter municipal véase J. SALAS, "Notas acerca de la Augustobriga vettona (actual Talavera la Vieja, Cáceres)", Norba, 6, 1985, pp. 57 ss.

14 J.A. Cean Bermúdez, Sumario de las Antigüedades Romanas que hay en España, Madrid, 1832, p. 425.

15 M. CORTÉs y LóPEZ, Diccionario geográfico-histórico de la España Antigua, T. II, Madrid, 1836, p. 184.

16 P. MADOz, Diccionario geográfico estadístico-histórico de España y sus posesiones de Ultramar, T. III, Madrid, 1847, p. 108. Véase también V. PAREDES, Origen del nombre de Extremadura, Plasencia, 1886, pp. 27 y 92.

17 ClL. II, 941: Flavia. Rufina. Augustobrig./annXXIII.h.s.e.s.t.t.I./ par.Honorina. et/ C. Rustenus.Decianus.filiae.fec; cf. también J. VIVES, Inscripciones latinas de la España romana, Barcelona, 1971, p. 483, n. 5255 .

18 Vid., MJSE, 29,1920, pp. 23 ss. No obstante de las excavaciones efectuadas hasta ahora en el yacimiento de Vascos, no se ha derivado aportación arqueológica romana de relieve que justifique tal identificación.

19 J.M. ROLDÁN, "Fuentes antiguas para el estudio de los vettones", Zephyrus, XIX-XX, 19689, pp. 89-90; Id., Iter ab Emerita..., op. cit., p. 158; Id., Itineraria Hispana..., op. cit., p. 220.

${ }_{20}$ CIL, II, 5346: C.lulius C.f.Gl[aber] / senatui popu[loque] / Augustobrilgensi] / hospes, d[onum] / dat; F. FITA, “Lápidas romanas inéditas", $B R A H, 11,1887$, p. 359, ofrece el siguiente 
venido identificando a Augustobriga con la citada localidad ya desde E. Hübner ${ }^{21}$, F. Fita ${ }^{22}$ y F. Coello ${ }^{23}$. Asimismo mantienen esta ubicación en Talavera la Vieja K. Miller ${ }^{24}$, A. García Bellido ${ }^{25}$, y A. Tovar ${ }^{26}$ entre otros; no obstante la distancia proporcionada por el Itinerario de Antonino de LV millas con respecto a Toletum, viene a cuestionar dicha tradicional identificación ${ }^{27}$, dificultando, por lo tanto, su definitiva y segura localización.

\section{Toletum}

Es mencionada también como mansio por el Itinerario de Antonino (Item a Liminio Toletum) a XLIIII m.p. de Consabro (It. Ant., 446, 7), así como por el Anónimo de Rávena $(312,10)$ con la forma de Toleton ${ }^{28}$. Por su parte Livio (XXXV, 7) hace referencia a Toletum, al relatar la victoria que M. Fulvio Nobilior llevaria a cabo en el 193 a.C., sobre una coalición de vacceos, vettones y celtiberos (maiores gestae res a M. Fulvio. is apud Toletum oppidum cum Vaccaeis Vectonibusque et Celtiberis signis collatis dimicavit. exercitum earum gentium fudit fugavitque, regem Hilernum vivum cepit) ${ }^{29}$. También Livio denomina a Toletum como parva urbs, sed loco munito $(X X X V, 22,5)$ en la narración del sitio y ocupación de la ciudad por M. Fulvio en el año 192 a.C. ${ }^{30}$, para posteriormente volver a referirse a

texto: C.lulius.C.f.Gal/senatui.popu / Augustobr / hospes.d/dat. En relación a los hallazgos y antigüedades de Talavera la Vieja puede verse entre otros: I. Hermosilla y SANDOVAL "Noticia de las ruinas de Talavera la Vieja", Memorias de la Acad. de la Historia, I, 1796, pp. 347-362; J. CORNIDE, "Continuación de la memoria de D.I. Hermosilla sobre las ruinas de Talavera la Vieja", Memoria de la Acad. de la Historia, I, 1796, pp. 363-408; A. PONZ, Viaje de España, vol. VII, Madrid, 1778, pp. 79 ss.; J.R. MELIDA, "Monumentos romanos de la antigua Augustóbriga", BRAH, 75, 1919, pp. 418-426; Id., Catálogo Monumental de España. Provincia de Cáceres (1914-1916), Madrid, 1924, pp. 88 ss.; F. JIMÉNEZ dE GRegorio, "Hallazgos arqueológicos en la Jara", AEArq., XXVIII, 1955, pp. 179 ss.; NAH, V, 1956-61, Madrid, 1962, pp. 235-7.

$21 \quad$ CIL, II, p. 831; RE II, 2, 1896, col. 2367.

22 F. FirA, “Lápidas romanas...", art. cit., p. 358.

${ }^{23}$ F. COELlo, "Vias romanas...", art. cit., pp. 5 ss.

24 Römische Reisewege..., op. cit., col. 151.

25 A. Garcia Bellido, La España del siglo primero de nuestra Era, Madrid, 1947, p. 252.

26 Iberische Landeskunde..., op. cit., p. 235.

27 La hipotética solución que se ha dado a la distancia ofrecida por el Itinerario entre Augustobriga y Toletum, es la posible omisión de una mansio intermedia correspondiente a Caesarobriga vid., nota n. ${ }^{\circ}$; G. ARIAS supone Augustobrigam-empalme en La Calzada de Oropesa, véase vgr., $M E, 24,1989$, p. 4 ; ME, 27,1990, p. 6; ME, 41,1992, p. 8.

28 Sobre el carácter del nombre, A. HolDER, Alt-Celtischer..., op. cit., vol. I1, p. 1871; también A. SCHULTEN, RE, VI, 2,1937, col. 1673.

29 FHA, III, p. 196.

sо FHA, III, p. 197; tum in Oretanos progressus et ibi duobus potitus oppidis Noliba et Cusibi ad Tagum amnem ire pergit. Toletum ibi parva urbs erat, sed loco munito. eam cum oppugnaret, 
este núcleo, al describir (XXXIX, 30) las campañas de los pretores del 186 a.C., L. Quinctio Crispino y C. Calpurnio Pisón sobre Carpetania y la derrota de los destacamentos militares romanos junto a Dipo y Toletum (eodem anno in Hispania praetores C. Calpurnius et L. Quinctius, cum primo vere ex hibernis copias eductas in Baeturia iunxissent, in Carpetaniam, ubi hostium castra erant, progressi sunt, communi animo consilioque parati rem gerere. haud procul Dipone et Toleto urbibus inter pabulatores pugna orta est. quibus dum utrimque subvenitur a castris, paulatim omnes copiae in aciem eductae sunt) ${ }^{31}$. Es citada igualmente por Ptolomeo ( $\left.T \omega \dot{\lambda} \eta \tau o v, l^{\prime} \mu \alpha^{\prime}\right)^{32}$ entre las ciudades carpetanas, así como por Plinio quien cataloga a los Toletani como estipendarios dentro del conventus Carthaginensis (Carpetaniae Toletani Tago flumini inpositi) ${ }^{33}$. Del testimonio epigráfico se puede deducir que llegaría a alcanzar organización municipal ${ }^{34}$, mostrando un desarrollo urbano atestiguado en los restos arqueológicos del circo ${ }^{35}$, acueducto ${ }^{36}$, puentes ${ }^{37}$, etc. Posteriormente adquiriría una notoria importancia en época visigoda.

Vectonum magnus exercitus Toletanis subsidio venit. cum is signis collatis prospere pugnavit et fusis Vectonibus operibus Toletum cepit.

3t FHA, III, p. 205.

32 Ptol., II, 6, 56. La forma Tád Parisiensis Supplem., 119, Parisiensis, 1402, Parisiensis, 1403, Parisiensis Coislin, 337, Vindobonensis, 1, Oxoniensis Seldanus, II, 46, Venetus, 516, Mediolanensis, D, 527, Vaticanus, 177, Vaticanus, 178, Vatic. Palatinus, 388, Vatic. Christinae reg. 82, S. Gregorii in monte Coelio, Florentinus Laurentianus, XXVIII, 49, Scorialensis $\Omega$, I, 1, Constantinopolitanus.

${ }_{33}$ Plin., NH, III, 25. Por su parte Grattio (Cyneg. 341) hace referencia al cultrum Toletanum, vid., FHA, VIII, p. 132 y A. SCHULTEN, Geografía y etnografía antiguas de la Peninsula Ibérica, II, Madrid, 1963, p. 334. Otras fuentes, lul. Honor., Cosmogr., p. 34,12: Toletum oppidum; Cosmogr. Aeth., p. 79, 13: Toletum, (Geographi Latini Minores, ed. A. Riese, Hildesheim, 1964).

${ }_{34}$ Vid., al respecto $\mathrm{M}^{\mathrm{a}} \mathrm{P}$. GonzALEZ-CONDE, Romanidad $e$ indigenismo en Carpetania, Alicante, 1987, pp. 57 ss.; G. AlFöldV, Römisches Städtewesen auf der neukastilischen Hochebene, Heidelberg, 1987, pp. 59-62; J. MANGAS, J. ALVAR, «La municipalización de Carpetania», Toledo y Carpetania en la Edad Antigua, Toledo, 1990, pp. 83 ss.; D. PLÁCIDO et alii, "Toletum", DArch., 1992, pp. 269 ss.

35 Véase M. CAStaños et alii, "Excavaciones en Toledo. Memoria de los trabajos efectuados en el circo romano", MJSEA, 96,1928; F. SAN ROMAN et alii, "Excavaciones en Toledo. Memoria de los trabajos efectuados en el circo romano", MISEA, 109,1930; A. REY PASTOR, El circo romano de Toledo, Toledo, 1932; FJ. WISEMAN, Roman Spain, London, 1956, p. 132; P. PIERNAVIEJA, "Los circos de Hispania", Segovia. Symposium de Arqueologia Romana, Barcelona, 1977, p. 319; J.H. HumpHREY, Roman Circuses. Arenas for Chariot racing, London, 1985, pp. 350-360; FJ. SÁNCHEZ-PALENCIA et alii, "Estratigrafia y arquitectura del circo romano de Toledo", I Congreso de $\mathrm{H}^{\mathrm{a}}$ de Castilla-La Mancha, T. IV, 1988, pp. 225-236; FJ. SANChiEZ-PALENCIA, M" J. SAINZ PASCUAL, El circo romano de Toledo: estratigrafía y arquitectura, Toledo, 1988; FJ. SÁNCHEZ-PALENCIA et alii, "El Circo romano de Toledo", Actas del Primer Congreso de Arqueología de la Provincia de Toledo, Toledo, 1990, pp. 351-369.

36 C. FeRnádez CASADO, Acueductos romanos en España, Madrid, 1972; Id., Ingenieria hidráulica romana, Madrid, 1983, pp. 514-515.

37 C. Fernández Casado, Historia del puente en España. Puentes romanos, Madrid, 1980. 
Resulta común su correspondencia con la actual Toledo, a cuyas antigüedades hiciera referencia Ambrosio de Morales ${ }^{38}$, y de la que proceden, por otra parte, diversos testimonios tanto epigráficos ${ }^{39}$ como numismáticos ${ }^{40}$. La misma identificación es unánimemente reconocida por $\mathrm{E}$. Flórez ${ }^{41}$, J. A. Cean Bermúdez ${ }^{42}$, M. Cortés y López ${ }^{43}$, E. Saavedra ${ }^{44}$, K. Miller ${ }^{45}$, A. Blázquez ${ }^{46}$, y más recientemente por $M$. Corchado Soriano ${ }^{47}$, J. M. Roldán Hervás ${ }^{48}$ y A. Tovar ${ }^{49}$, entre otros muchos ${ }^{50}$.

\subsection{Per Lusitaniam ab Emerita Caesarea Augusta}

Es denominada por Wesseling Per Lusitaniam ab Emerita Caesaraugusta, y por E. Saavedra ${ }^{51}$ con el número 29 Per Lusitaniam ab Emerita Caesaraugustam. Con un total de CCCCLVIII m.p., no constituía frente a la ruta anterior (It. Ant., 438, 2, ss.), la vía de comunicación más directa entre Emerita y Caesaraugusta. En su trayectoria desde el SW al NE, presenta una serie de tramos claramente diferenciados; en primer término de Emerita a Laminio, de Laminio a Titulcia, y un último de Titulcia a Caesaraugusta común a otras vías ${ }^{52}$. Por otro lado la referencia expresa de per Lusitaniam a una vía que tan rápidamente deja el área propiamente

${ }^{38}$ Ambrosio DE Morales, Las Antigüedades de las ciudades de España, Alcalá de Henares, MDLXXV, pp. 90 y ss.

39 Véase vgr., ClL, II, pp. 416 ss.

40 Vid., A. VIVES, La moneda hispánica, Madrid, 1924-6, lám. CXXXIV; O. Gil FARRES, La moneda hispánica en la Edad Antigua, Madrid, 1966, pp. 183,186-88, 252 ss. y 274; A.M. GUADAN, Numismática ibérica e ibero-romana, Madrid, 1969, p. 210; L. VILLARONGA, Numismática antigua de Hispania, Barcelona, 1979, pp. 229, 241-2.

41 E. Flórez, España Sagrada, V, pp. 173 ss.

42 Sumario de las Antigüedades..., op. cit., pp. 118-119.

43 Diccionario geográfico-histórico..., op. cit., vol. III, pp. 421-3.

44 Discursos..., op. cit., p. 104.

45 Römische Reisewege..., op. cit., col. 151 y 163.

46 MJSEA, 29, 1920, pp. 23 ss.

47 M. CORCHADO SORIANO, "Estudio sobre vias romanas entre el Tajo y el Guadalquivir", AEArq., 42,1969, p. 156.

48 Itineraria Hispana..., op. cit., p. 272.

49 A. Tovar, Iberische Landeskunde. Las tribus y las ciudades de la antigua Hispania, 3, Tarraconensis, Baden-Baden, 1989, pp. 229-232.

50 Según M. Fennandez Miranda et alii, "Indigenismo...", ant. cit., p. 17, Toletum mansio se situaría en las inmediaciones de la ciudad romana del mismo nombre; G. Arias por su parte, coloca Toletum-empalme en "Portillo de Toledo", vid., ME, 27,1990, p. 6, y ME, 41, 1992, p. 14.

51 Discursos..., op. cit., p. 76.

52 Véase It. Ant., 436, 1-438, $1 ; 438,8-439,4 ; 439,11-14$. A este último tramo de Titulcia a Caesaragusta, se hace mención al igual que en 439,13-14 con la referencia Caesaraugusta mansionibus supra scriptis, dándose la cifra de CCXV m.p. 
lusitana, por supuesto mucho antes que It. Ant., 433, 1 ss., ha venido a plantear diversos problemas de interpretación para con esta ruta ${ }^{53}$, que además y según J. M. Roldán ${ }^{54}$ coincidiría en su primera parte con el ltem a Corduba Emeritam (It. Ant., 415, 3-416, 3).

El desarrollo de esta via ${ }^{55}$ hasta el ámbito objeto de estudio, es descrita en la edición manejada ${ }^{56}$, de la forma siguiente:

\begin{tabular}{|c|c|c|c|}
\hline 444 & 3 & $\begin{array}{l}\text { Per Lusitaniam } \\
\text { ab Emerita } \\
\text { Caesarea }\end{array}$ & \\
\hline \multirow{10}{*}{445} & 4 & Augusta & $\begin{array}{c}\text { m.p. CCCCLVIII, } \\
\text { sic: }\end{array}$ \\
\hline & 5 & Contosolia & m.p. XII ${ }^{57}$ \\
\hline & 6 & Mirobriga & m.p. $X X X V I$ \\
\hline & 7 & Sisalone & m.p. XIII \\
\hline & 1 & Carcuvium & m.p. XX \\
\hline & 2 & Ad Turres & m.p. XXVI \\
\hline & 3 & Mariana & m.p. XXIIII \\
\hline & 4 & Lamini & m.p. $X X X$ \\
\hline & 5 & Alces & m.p. XL \\
\hline & 6 & Vico Cuminario & m.p. XXIIII \\
\hline
\end{tabular}

53 Se ha apuntado incluso la posible confusión de per Lusitaniam por Laminium, vid., F. COELLo, "Vias romanas...", art. cit., p. 18. También y en cuanto a la interpretación del término per Lusitaniam en relación a esta via, véase G. ARIAS, Repertorio..., op. cit., pp. 510-11, y ME, 2, 1963 , p. 32; ME, 13, 1967, p. 374; ME, 34, 1991, p. 11; ME, 39, 1992, pp. 14-15.

${ }^{54}$ J. M. ROLDÁ, Iter ab Emerita..., op. cit, p. 154; también ya al respecto V. PAAEdes, Origen del nombre de Extremadura, op. cit., p. 96.

${ }_{55}$ Véase en general: F. COELLO, "Vias romanas...", art. cit., BRAH, XV, 1889; A. BLAZQUeZ, "Vias romanas de la Beturia de los Turdulos", BRAH, 61,1912, pp. 359 ss.; MJSEA, 9, 1917; MJSEA, 40,1921; ME, 2,1963; ME, 13,1967; M. CORCHAdO SORIANO, "Estudio sobre vías...", art. cit., AEArq, 42,1969; J. M. RolDÁN, Iter ab Emerita..., op. cit., p. 154; Id., Itineraria Hispana..., op. cit., pp. 91-3; G. ARIAS, Repertorio..., op. cit., pp. 101-2 y 510-11; ME, 18,1988; ME, 21,1989; ME, 23,1989; $M E, 25,1990 ; M E, 34,1991 ; M E, 36,1992 ; M E, 39-41,1992$.

$56 \quad$ Itineraria Romana..., op. cit., vid. nota 8 ; en esta edición se hace constar en el aparato critico: 444,3 lysitaniam $L ; 6$ mirobrica $B ; 445,6$ cominario $B$.

${ }_{57}$ E. SaAvedra, Discursos..., op. cit., p. 76, proporciona la distancia de mpm XV. 


\section{MANSIONES}

\section{Vico Cuminario}

Es mencionada como mansio en esta vía del Itinerario, situada entre Alces ${ }^{58}$ de la que la separa XXIIII m.p., y Titulciam distante XVIII m.p. De localización imprecisa, sería no obstante ubicada por Ambrosio de Morales ${ }^{59}$ en Santa Cruz de la Zarza, de la misma manera que $E$. Flórez ${ }^{60}$ y J. A. Cean Bermúdez ${ }^{61}$; también A. Laborde en su Itineraire descriptif de l'Espagne ${ }^{62}$, recoge esta misma creencia, aún cuando para A. Blázquez ${ }^{63}$ se situaria en La Guardia. M. Cortés y López ${ }^{64}$, sin embargo, frente a los criterios anteriores mencionados, estimaría más conveniente colocar el emplazamiento de esta mansio en la localidad de Ocaña, opinión seguida igualmente por P. Madoz ${ }^{65}$. Por su parte Eduardo Saavedra ${ }^{66}$ se inclinaría por fijar la situación de Vico Cuminario entre Lillo y La Guardia, hipótesis mantenida de forma análoga por Aureliano Fernández-Guerra ${ }^{67}$. Más recientemente tanto $A$. Schulten ${ }^{68}$ como $A$. Tovar ${ }^{69}$ sin especificar lugar concreto, vienen a suponerla al Este de Toledo, mientras que J.M. Roldán Hervás ${ }^{70}$ reconoce no estar asegurada su exacta localización.

58 La no asegurada localización de Alces, impide se inscriba con absoluta certeza en el área geográfica que nos ocupa, así pues mientras que para E. SAAVEDRA, Discursos..., op. cit., p. 84, se ubicaría al O. de Miguel ESTEBAN, y para A. BlÁzQUEZ, MJSEA, 9,1917 y 40,1921 en las imnediaciones de la Hidalga entre Campo de Criptana y Quero, sin embargo según A. FERNANDEZGUERRA, M. CORTES Y LOPEZ y P. MADOz entre otros, se situaria en la localidad de Alcázar de San Juan. Según J. M. ROLDÁN, no existe seguridad en su identificación con la Alce de que habla $T$. Livio $\mathrm{XL}, 48$ : Inde iam duxit ad Alcen urbem... y XL, 49: convertit inde agmen retro unde venerat ad Alcen atque eam urbem oppugnaret institit; véase sin embargo al respecto $R E, 1,1893$, col. 1338.

59 Las Antigüedades de las ciudades..., op. cit., pp. 76-7; Ambrosio DE MORALEs relaciona el nombre de esta mansio al "Cuminum" tan alabado por Plinio en XIX, 161 para la zona de Carpetania: in Carpetaniam nostri orbis laudatur cuminum; véase también en este mismo sentido,

A. SCHULTEN, Geografia y etnografia antiguas..., op. cit., vol. II, p. 417.

6o Vid., España Sagrada, V, pp. 22-23.

61 Sumario de las Antigüedades..., op. cit., pp. 111-112.

62 A. LABORDE, Itineraire descriptif de l'Espagne, vol. IV, París, $1828^{3}$, p. 7.

63 MJSEA, 40, 1920-1.

64 Diccionario geográfico-histórico..., op. cit., vol. III, p. 490; también y más recientemente sobre la hipótesis de Ocaña vid., ME, 23,1989.

65 P. MADoz, Diccionario geográfico-estadistico-histórico..., op. cit., vol. XVI, p. 23

66 Discursos..., op. cit., p. 106.

67 Vid., BRAH, I, 1877, p. 137.

68 A. SCHULtEN, Geografia y etnografía antiguas..., op. cit., vol. II, p. 417.

${ }_{69}$ A. Tovar, Iberische Landeskunde..., op. cit., vol. III, p. 237.

70 Itineraria Hispana..., op. cit., p. 278. 


\subsection{Item a Liminio Toletum}

E. Saavedra con el número 30 la designa Item a Laminio Toletum ${ }^{71}$, corrigiendo de la misma manera que el resto de los autores Liminio por Laminio ${ }^{72}$. Viene a constituir sin duda, una de las vías más breves de las descritas por el Itinerario de Antonino, siendo su longitud total asignada de XCV m.p. Las mayores dificultades que ofrece su trazado están en relación, y como bien apunta J. M. Roldán, con las distancias entre las diversas mansiones, junto y una vez más a la problemática de la localización de éstas ${ }^{73}$.

En la edición utilizada ${ }^{74}$, esta vía en su totalidad es descrita de la siguiente forma:

$\begin{array}{rll}\text { 446, } 4 & \text { Item a Liminio } \\ & \text { Toletum } & \text { m.p. XCV, sic: } \\ 5 & \text { Murum } & \text { m.p. XXVII } \\ 6 & \text { Consabro } & \text { m.p. XXIIII } \\ 75 & \text { Toletum } & \text { m.p. XLIIII }\end{array}$

71 E. SaAvedra, Discursos..., op. cit., p. 76.

72 Véase vgr., P. Wesseling: Iter a Laminio Toletum; en el mismo sentido G. PArther et $M$. PINDER, Itinerarium..., op. cit., p. 213, y J. M. ROLDÁ, Itineraria Hispana..., op. cit., p. 94. Vid., no obstante al respecto G. ARIAS, ME, 11,1966, pp. 289-90, y también Id., Repertorio..., op. cit., pp. 144-8.

${ }_{73}$ En relación a esta vla, véase: MJSEA, 9,1917; ME, 3,1963; ME, 10, 1965; ME, 11.1966; M. CoRchado Soriano, “Estudio sobre vias...", art. cit., AEArq., 42, 1969; J. M. ROLDÁN, Itineraria Hispana..., op. cit., pp. 93-4; G. ARIAS, Repertorio..., op. cit., pp. 137 y $144-$ 8; ME, 16,1988; C. FERNÁNDEZ et alii, "Entre Consabro y Laminio: aproximación a la proble. mática de la vía 30 del Itinerario". Simposio sobre la red viaria en la Hispania Romana, Zaragoza, 1990, pp. 165-182; $M E, 25,1990 ; M E, 26,1990 ; M E, 27,1990 ; M E, 35,1991 ; M E, 39-$ 41, 1992.

${ }_{74}$ Itineraria Romana..., op. cit., vid. nota 8; en el aparato crítico de dicha edición se hace constar: 446,4 R III super sunt in marg. B; 5 VII $B$ XXVII R; 6 XXVIII B; 7 R hic IIII super sunt in marg. $B ;$ P. WESSELING ofrece el desarrollo de esta vía de la siguiente forma:

$\begin{array}{ll}\text { Iter a Laminio Toletum } & \text { m.p. XCV sic. } \\ \text { Murum } & \text { m.p. XXVII } \\ \text { Consabro } & \text { m.p. XXVIII } \\ \text { Toletum } & \text { m.p. XL }\end{array}$

75 G. Parthey et M. Pinder, Itinerarium..., op. cit., p. 213, y E. SaAvedra, Discursos..., op. cit., p. 76 proporcionan la distancia de $\mathrm{mpm}$ XXVIII. 


\section{MANSIONES}

\section{Consabro}

Es citada además de por el Itinerario de Antonino en esta vía, entre Murum y Toletum distante XLIIII m.p., por el Anónimo de Rávena (313, 15) con el nombre de Consabron ${ }^{76}$. También y en Ps. Frontino IV, 5,19 (Hispani Consabrae obsessi eadem omnia passi sunt nec oppidum Hirtuleio tradiderunt) se hace referencia a este núcleo con motivo de las operaciones militares durante las guerras sertorianas en el año 79 a.C. ${ }^{77}$. Plinio por su parte, incluye entre las comunidades estipendarias pertenecientes al conventus Carthaginensis a los Consaburrenses (stipendiariorum autem celeberrimi..., Consaburrenses,... ${ }^{78}$. Además, y según el testimonio de la inscripción honorifica (CIL, II, 4211) dedicada a L. Domitius $M$. fil. Serg. Dentonianus, quien ostenta el cargo entre otros de duumviro del municipium de Consabura ${ }^{79}$, queda claramente constatado su status municipal ${ }^{80}$.

Existe unanimidad en su identificación con la localidad de Consuegra, de cuyos vestigios romanos dejara ya testimonio $\mathrm{D}$. Aguirre en el siglo XVIII ${ }^{81}$. Análoga correspondencia es igualmente llevada a cabo por J.A. Cean Bermúdez ${ }^{82}$, M. Cortés y López ${ }^{83}$ y P. Madoz ${ }^{84}$;

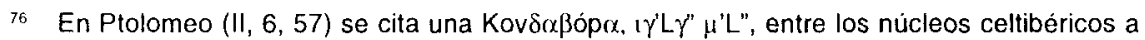
continuación de Segóbriga; sobre su identificación con Consabura vid., A. Tovar, Iberische Landeskunde..., op. cit., vol. III, pp. 222-223.

77 Vid., FHA, IV, p. 177.

78 Plin., NH, III, 25. Consaburensis se atestigua epigráficamente en $C / L, 11,2166: Q$. Domitius / Q.L. Macer / Consaburensis / L.p. in fronte XV/ in agro $p$. $X X$; vid., también J. Vives, Inscripciones..., op. cit., p. $487, n^{\circ} 5305$.

${ }_{79}$ CIL, II, 4211: P.H.C / L. Domitio / M. fil. Serg. / Dentoniano / iudic. dec. V equo / publico per Traian. / II vir. munic Consaburon / flam[i]n. perpet. tribun. milit. / cohort. Astur. Gallaeciae / et Mauretan. Tingit. / flam. P.H.C.; H. DESSAU, ILS, II, p. 691, $n^{\circ} 6936$; J. VIVES, Inscripciones..., op. cit., p. 185, $n^{\circ}$ 1586; G. AlföldY, Flamines Provinciae Hispaniae Citerioris, Madrid, 1973, p. 69, $n^{\circ}$ 20; Id., Die römischen Inschriften von Tarraco, Berlín, 1975, p. 149, $n^{\circ} 271$. En relación a la tribu Sergia de Dentonianus vid., W. KuBITSCHEk, De romanorum tribuum origine ac propagatione, Wien, 1882, $\mathrm{p}$. 171; Id., Imperium romanorum tributim descriptum, Wien, 1889, p. 193; R. WIEGELS, Die Tribusinschriften..., op. cit., pp. 108-9; G. ALFöLDY, Römisches Städtewesen..., op. cit., pp. 53-4.

so Véase RE, IV, 1, 1900, col. 889; H. Galsterer, Untersuchungen, op. cit., p. 70; M P. GonzALEZCONDE, Romanidad..., op. cit., pp. 89 ss.; Id., "L. Domitius Dentonianus y la promoción jurídica de Consabura", HAnt., XIII, 1986-9, pp. 53-70; G. AlföloY, Römisches Städtewesen..., op. cit., pp. 52-54.

81 D. AguirRe, El Gran Priorato de San Juan de Jerusalén en Consuegra, en 1769. Toledo, 1973.

92 Sumario de las Antigüedades..., op. cit., p. 71.

83 Diccionario geografico-histórico..., op. cit., vol. 11, pp. 378-9.

${ }^{84}$ P. MADOZ, Diccionario geográfico-estadístico-histórico..., op. cit., vol. VI, p. 569. 
también realizan dicha equivalencia $E$. Saavedra ${ }^{85}$, E. Hubner ${ }^{86}$ y $A$. Fernández-Guerra ${ }^{87}$, así como y entre otros $\mathrm{K} \mathrm{Miller}^{88}$, $\mathrm{A}$. Blázquez ${ }^{89}$, A. Garcia Bellido ${ }^{90}$, M. Corchado Soriano ${ }^{91}$, J.M. Roldán Hervás ${ }^{92}$ y A. Tovar ${ }^{93}$. Diversos restos y hallazgos arqueológicos ${ }^{94}$, vienen, por otra parte, a confirmar dicha localización, a la vez que atestiguan la entidad que, paralela a su municipalización, llegaría a alcanzar este núcleo.

\section{ANÓNIMO DE RÁVENA}

Junto al Itinerario de Antonino, el Ravennatis Anonymi Cosmographia o vulgarmente Anónimo de Rávena, viene a representar otra de las fuentes itinerarias antiguas a tener en cuenta para el ámbito que nos ocupa. Datada en el siglo VII ${ }^{95}$, no proporciona, no obstante, las distancias entre una mansión y otra, limitándose solamente a consignar el nombre de éstas.

En la edición manejada ${ }^{96}$, los recorridos contenidos en esta obra, referentes al área geográfica objeto de estudio y zona limítrofe, se describen de la manera siguiente:

85 Discursos..., op. cit., p. 91.

86 CIL, II, p. 431.

$87 \quad B R A H, \mathrm{l}, 1877$, p. 137.

88 Römische Reisewege..., op. cit., col. 163.

89 MJSEA, 9, 1917, pp. 19 ss.

90 A. Garcia Bellido, La España del siglo primero..., op. cit., p. 238.

91 M. CoRchado Soriano, “Estudio sobre vías...", art. cit., p. 151.

92 Itineraria Hispana..., op. cit., p. 233.

93 Iberische Landeskunde..., op. cit., vol. III, pp. 222-4.

94 Véase al respecto, J. JIMÉNEZ DE GREGORIO, "Hallazgos arqueológicos en la provincia de Toledo", AEArq., 36,1963, pp. 228 ss.; FJ. GILES PACHECo, "Contribución al estudio de la arqueología toledana. Hallazgos hispanorromanos en Consuegra", Anales Toledanos, 5 , 1971, pp. 139-165; J.A. GaACiA-DiEgo, "Una presa romana en Consuegra", Agua, 90, 1975, pp. 2-9; J. A. GARCIA-DIEGo et alii, "Nuevo estudio sobre la presa romana de Consuegra", Rev. de Obras Públicas, 1980, pp. 487-505; C. FERnANDEZ CASADO, Ingenieria hidráulica.... op. cit., p. 147; J. A. GARCIA-DIEGo et alii, “Estudio conjunto sobre la presa romana de Consuegra", Rev. de Obras Públicas, 1983, pp. 673-688; J.C. FERnÁndez-LAyos, $H^{a}$ de Consuegra, I. Edad Antigua, Toledo, 1983, pp. 98 ss.; A. BLANCO FrEJEIRO, "Presa romana de Consuegra (Toledo). Declaración de monumento histórico-artístico", BRAH, CLXXX, 1983, pp. 191-2.

${ }_{95}$ Para cuestiones de cronologia y carácter de esta obra vid., J.M. RoLDÁN, Itineraria Hispana..., op. cit., pp. 111 ss.

${ }_{96}$ Ravennatis Anonymi Cosmographia et Guidonis Geographica. Ed. M. Pinder et G. Parthey, Aalen, 1860 (Reed. 1962). 
44. Iterum quomodo in media provincia ipsius patriae Spaniae dicitur civitas

$\begin{aligned} 7 & \text { Complutum, cuius affinalis est } \\ 8 & \text { civitas quae dicitur } \\ 9 & \text { Titultia, item civitas } \\ 10 & \text { Toleton } \\ 11 & \text { Lebura } \\ 12 & \text { Augustabria }\end{aligned}$

Se trata de una parte del trazado de la ruta de Complutum a Emerita (It. Ant., 438, 2-438, 9), en el que se incluye una nueva mansión, Lebura, no citada por el Itinerario de Antonino para este tramo.

\section{MANSIONES}

\section{Lebura}

Situada entre Toleton y Augustabria (Augustobriga vid., supra) por el Ravennate, en Ptolomeo (II, 6, 56) se menciona $\Lambda$ ı $\beta o ́ \rho \alpha, \theta^{\prime} \gamma^{\prime} 1 \beta^{\prime \prime} \mu^{\prime} L^{\prime \prime} \delta^{\prime \prime} 97$ entre los núcleos de población carpetanos. No es segura su identificación con la Aebura mencionada por Livio ${ }^{98}$ al narrar las campañas de $\mathrm{Q}$. Fulvius Flaccus en el año 181 a.C. No obstante para A. Schulten ${ }^{99}$ dicha equivalencia sería factible; $V$. Paredes ${ }^{100}$ y K Miller ${ }^{101}$ la localizan en

${ }^{97}$ La forma $\Lambda 1 \beta \omega \rho \alpha$ se ofrece en los códices siguientes: Parisiensis Supplem., 119, Venetus, 383, Venetus, 516, Vaticanus, 178; $\wedge 1 \beta \alpha \rho \alpha$ en el Parisiensis, 1404, Parisiensis, 1403, y Vatic. Palatinus, 314. Variantes en las graduaciones: $\theta^{\prime} \gamma^{\prime \prime} \beta^{\prime \prime}$ en el Vaticanus, 191, Florentinus Laurentianus, XXVIII, 9, Florentinus Laurentianus, XXVIII, 38, Florentinus Laurentianus, XXVIII, 42. $\theta^{\prime} \gamma$ 'ro" en Parisiensis, 1404, Parisiensis, 1403, Parisiensis, 2423, Vatic. Palatinus, 314. $\theta$ ' $\mathrm{O}^{\prime \prime}$ en otros. $\mu \delta \delta^{\prime} L^{\prime \prime} \delta$ en Florentinus Laurentianus, XXVIII, 9, Florentinus Laurentianus, XXVIII, 38, y Florentinus Laurentianus, XXVIII, 42.

98 Liv. XL, 30, 3-4: principio veris exercitum in Carpetaniam duxit et castra locavit ad oppidum Aeburam modico praesidio in urbe posito. paucis post diebus celtiberi milia duo ferme inde sub colle posuerunt castra. $\mathrm{XL}, 32,5$ : simul ab urbe Aebura, qui in praesidio relicti erant in medio ardore pugnae advenerunt et Acilius ab tergo erat $\mathrm{XL}, 33,1$ : sauciis deinde in oppidum Aeburam devectis per Carpetaniam ad Contrebiam ductae legiones. Cf. FHA, III, pp. 211-212.

99 Vid., RE, XIII, 1,1926, col. 116; FHA, III, p. 212

100 V. Paredes, op. cit., p. 27.

101 Römische Reisewege..., op. cit., col. 151. 
Talavera de la Reina ${ }^{102}$, sin embargo para F. Fita ${ }^{103}$ "debió hallarse al otro lado del Tajo..., y por consiguiente hay que buscarla siguiendo la calzada o camino romano que pasa por el puerto Marchés». Por su parte $P$. Boch-Gimpera y $P$. Aguado Bleye recogiendo la opinión que identifica Aebura y Libora, la sitúan en Puebla de Montalbán ${ }^{104}$. Más recientemente A. Tovar ${ }^{105}$ descartando una posible equivalencia Aebura/Libora, sitúa a esta última cerca del limite entre la Tarraconense y la Lusitania, sin precisar lugar exacto, constatando de esta forma su dificultosa identificación.

IV 44

8

9

15

16

17
Iterum iuxta ipsam civitatem Complutum est civitas quae dicitur

Consabron

Moroin

Lamini

Viene a constituir parte de la ruta ya descrita por el Itinerario de Antonino, Item a Liminio Toletum (It. Ant., 446, 4-7), experimentándose solamente algunas modificaciones en los nombres de las respectivas mansiones, como Consabron por Consabro, Moroin por Murum, y Lamini por Laminio.

A estas vias mencionadas en las antiguas fuentes itinerarias, habrá que añadir otras rutas no atestiguadas en dichas fuentes, y que podrán ser analizadas mediante la correspondiente labor arqueológica, en un ámbito territorial como éste, de gran interés en el trazado de las comunicaciones romanas de la Meseta.

102 Véase también al respecto y más recientemente, $M E, 27,1990$, p. 6; ME, 36, 1992, p. 23; $M E, 41,1992$, p. 11.

103 BRAH, 2, 1882, p. 297.

104 Vid., Ha de España de R. MENEndez PIDAl, II, Madrid, 1962, p. 75; véase también comentario de C. Müller, ad Ptol., I, p. 176: "Libora sec. Ptol. et Rav. inter Toletum et Augustobrigam (Talavera de la Reina) sita fortasse stetit ubi nunc est Montalba ad Tagum fl". De forma análoga Chr. Gouillart, T. Live. Histoire Romaine, T. XXX, Paris, Les Belles Lettres, 1986, p. 112, nota 5.

105 Iberische Landeskunde..., op. cit., vol. III, pp. 235 y 232-3. 


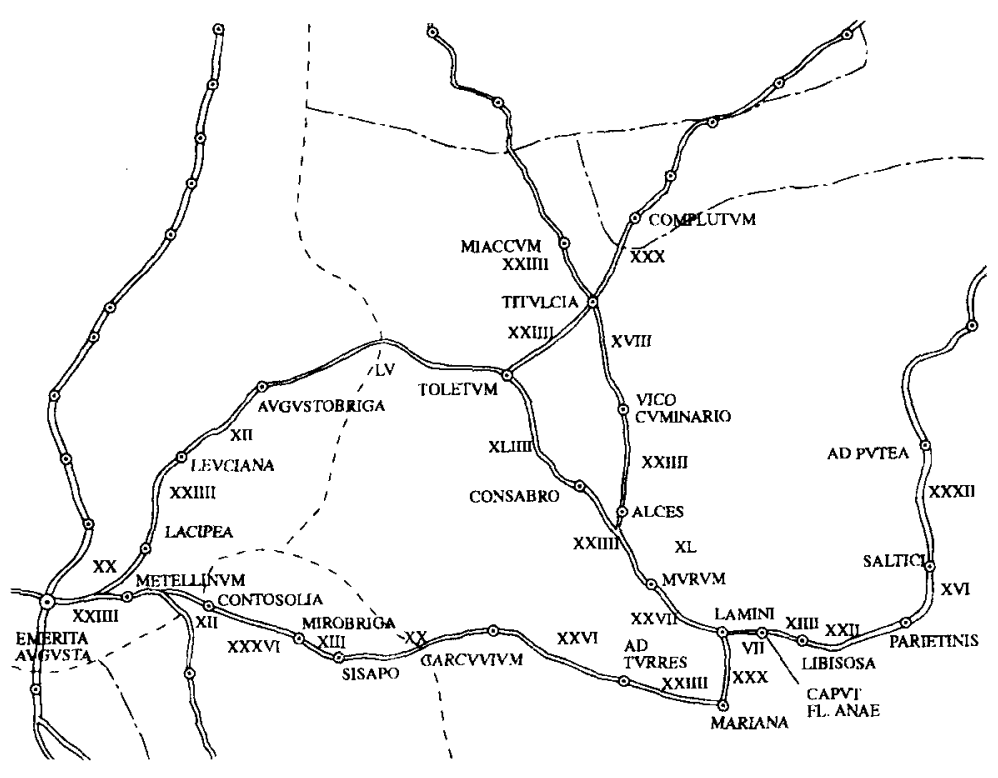

Figura 1. Vias romanas de la Meseta sur y áreas circundantes en el litinerario de Antonino, según J.M. Roldán.

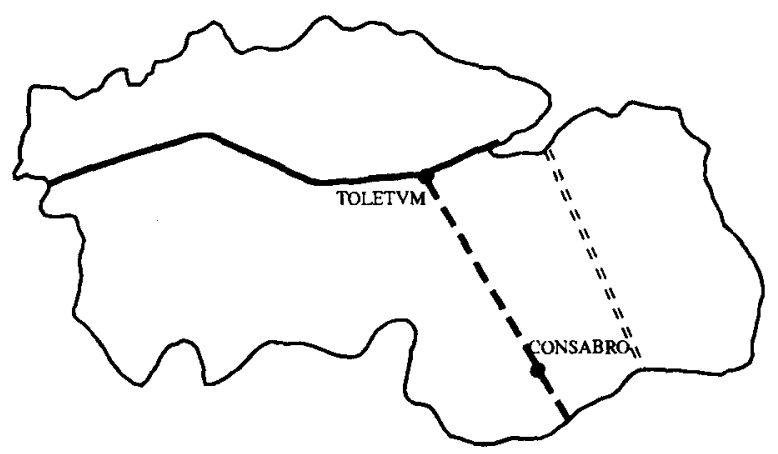

Via 25, Alio itinere ab Emerita Caesarea Augusta

$\Rightarrow=$ : $=$ Via 29. Per Lusitaniam ab Emerita Caesarea Augusta

... Via 30, Item a Laminio Toletum

Figura 2. Vías romanas de la provincia de Toledo atestiguadas en el Itinerario de Antonino. 